\title{
Development of Quantitative Techniques with Time-of-Flight Secondary Ion Mass Spectrometry (TOF-SIMS) for Li Characterization in High Energy Batteries.
}

\author{
S. Bessette ${ }^{1,2}$, P. Hovington ${ }^{1}$, C. Kim ${ }^{1}$, R. Gauvin ${ }^{2}$, H. Demers ${ }^{2}$ and K. Zaghib ${ }^{1}$. \\ 1. Hydro-Quebec's Research Institute, Varennes, Quebec, Canada. \\ 2. Department of Mining and Materials Engineering, McGill University, Montreal, Quebec, Canada.
}

Energy dispersive $\mathrm{x}$-ray spectroscopy (EDS) is the standard technique when it comes to material characterization with scanning electron microscopy (SEM). However, for lithium related materials, even a windowless EDS detector with optimized electronics will have limitations in the detection and quantification of Li content due to low sensitivity (>20 wt\%) and lack of understanding of Li x-ray emission processes [1]. TOF-SIMS in a focused ion beam scanning electron microscope (FIB-SEM) allows imaging with a resolution greater than $50 \mathrm{~nm}$ and high sensitivity for $\mathrm{Li} \mathrm{(>2} \mathrm{ppm} \mathrm{[2]).} \mathrm{However,}$ quantification using TOF-SIMS is difficult, mainly due to matrix effects and the change of sputtering yield with primary beam angle incidence (edge effects). Quantification models already exist for TOFSIMS using secondary implanted standards [3], however those are developed for trace elemental analysis of implants in homogenous, simple structures. Battery related materials have high elemental concentrations with complex stoichiometry. Since Li is the key element in battery materials and the sputtering yield is very high for Li (very high sensitivity), a quantitative model must be developed with TOF-SIMS that can be applied to electrode materials, as well as lithium metal and solid electrolyte interface (SEI).

In this work, experiments will be made first on standard lithium nickel cobalt manganese oxide (NCM) cathodes using a FIB-SEM TESCAN Lyra3 microscope equipped with a TOF-SIMS analyzer from the collaborative work of TESCAN and TOFWERK AG. A primary $\mathrm{Ga}^{+}$beam with a landing energy of 30 $\mathrm{kV}$ was used for all the experiments. Samples were prepared using an IM4000PLUS ion milling system from Hitachi High Technologies.

Figure 1 shows an example of a TOF-SIMS acquisition on standard NCM particle for $\mathrm{Li}^{7}$. Signal variations are seen on the particle and at interfaces with the porous medium. For quantification purposes, true signal variations must be distinguished from variations due to edges effect. In order to minimize those effects, samples have been prepared with the ion milling system to ensure a flat surface for analysis.

Figure 2 shows the TOF-SIMS recorded intensities for active battery materials on standard NCM cathodes with different degree of lithium insertion $(x)$. According to the results, Li intensity decreases with decreasing $x$ values. However, the decrease in Li intensity from $x=1$ to $x=0.25$, about $30 \%$, does not correlate with the theoretical decrease of $\mathrm{Li}$ content in $\mathrm{Li}_{x} \mathrm{Ni}_{a} \mathrm{Co}_{b} \mathrm{Mn}_{c} \mathrm{O}_{2}$ compound, which is more than twice the intensity drop observed with TOF-SIMS. The same correlation can be done for $\mathrm{Mn}, \mathrm{Ni}$ and Co, where the increase in content is not directly correlated to the TOF-SIMS intensities increase. These signal variations clearly show that some matrix effects are occurring. Preferential sputtering [3] can also emphasize Li sputtering, leading to increased intensity recorded for low x values.

In order to determine true elemental concentration, relative sensitivity factors (RSF), which are intensity correction factors, are usually used to take account for the environment in which the species of interest is found (matrix effect). RSF reflect the sensibility of an ion in a specific matrix under ion bombardment. 
Figure 3 shows preliminary RSF values obtained for $\mathrm{Li}, \mathrm{Mn}, \mathrm{Ni}, \mathrm{Co}$ in the cathodes. Results follow the general trend of RSF values found in the literature [4] although these are usually obtained for ion implanted in $\mathrm{Si}$ and under oxygen primary beam. At lower $\mathrm{Li}$ content $(x=0.25)$ the RSF value is significantly lower which could show a stronger matrix effect for this material. More work will be done to confirm these results and also confirm the Li concentration using another analytical technique.

In conclusion, TOF-SIMS is a promising technique for Li quantification because of its high sensitivity, but has to be optimized for battery applications.

\section{References:}

[1] P. Hovington et al, Scanning, Vol. 38, Nov/Dec 2016, pp. 571-578

[2] F. A. Stevie et al, Surface and Interface Analysis, SIMS proceedings paper, 2014.

[3] F.A. Stevie, Secondary ion mass spectrometry: Applications for depth profiling and surface characterization. New York: Momentum Press, 2016.

[4] R.G. Wilson, F.A. Stevie and C.W. Magee. "Secondary ion mass spectrometry",1.6-10. New York: Wiley 1989.

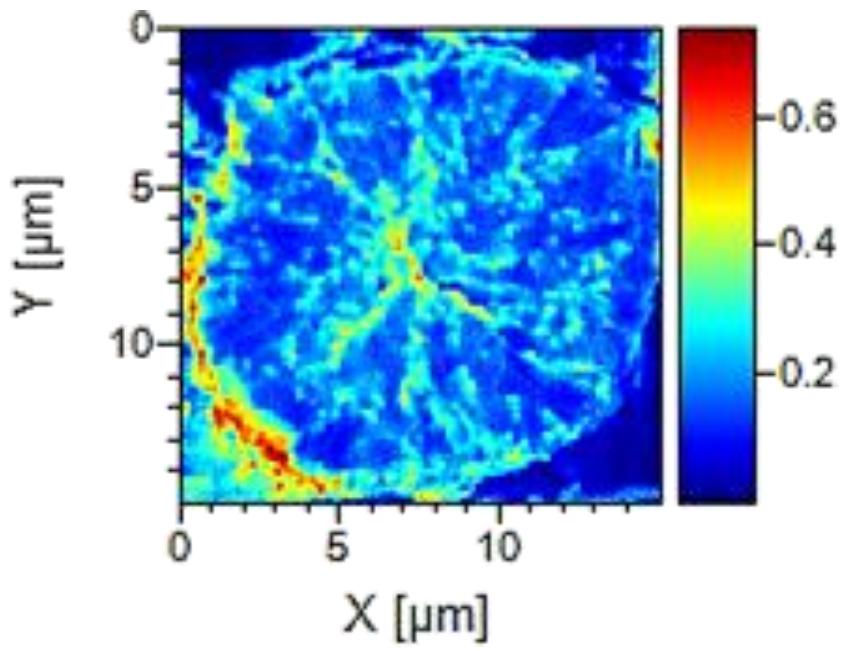

Figure 1. $\mathrm{Li}^{7}$ mapping using TOF-SIMS detector with $460 \mathrm{pA}, 30 \mathrm{kV}$ Ga+ beam on NMC particle.

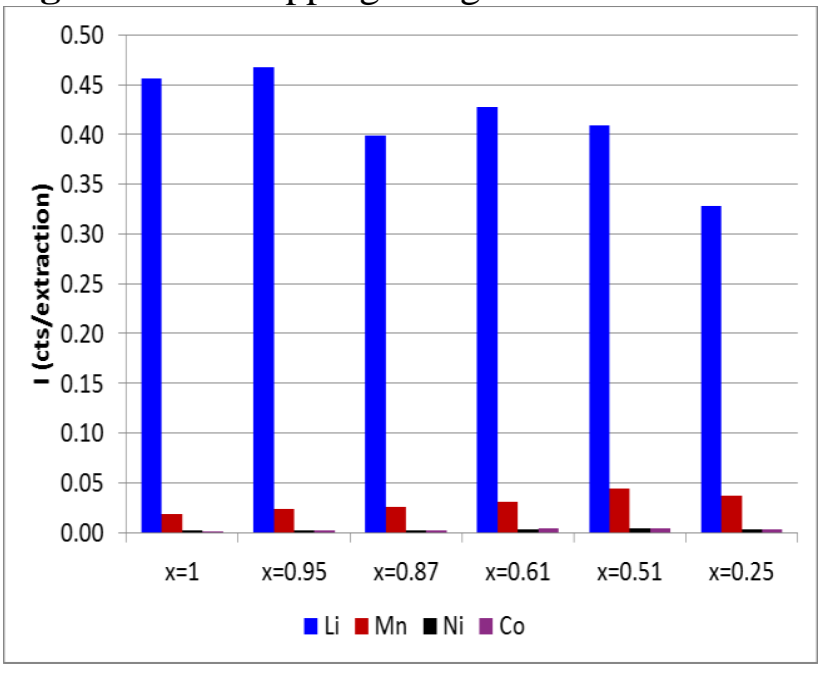

Figure 2. TOF-SIMS intensities of active elements in cathodes of $\mathrm{Li}_{x} \mathrm{Ni}_{a} \mathrm{Co}_{b} \mathrm{Mn}_{c} \mathrm{O}_{2}$ with varying $\mathrm{x}$.

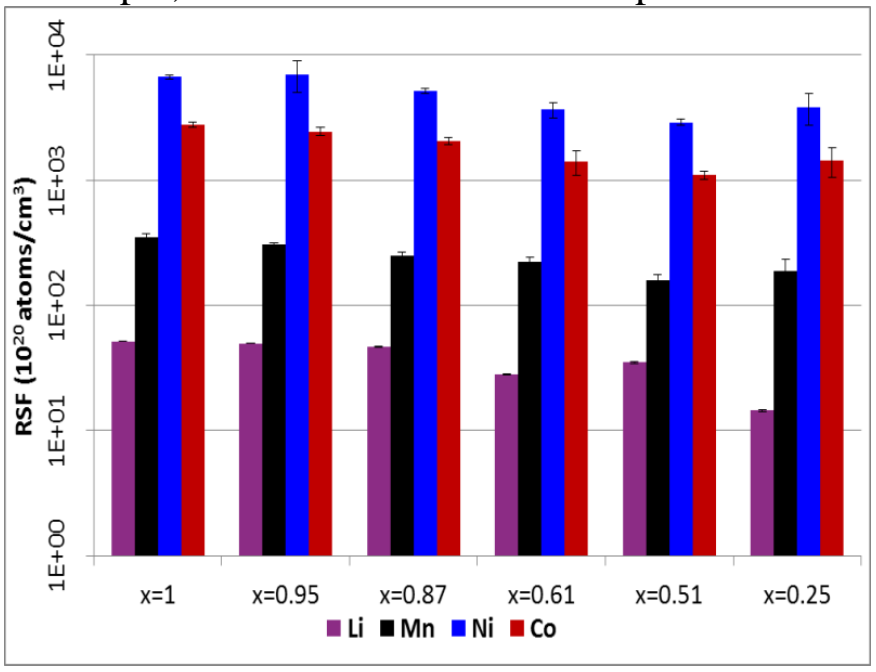

Figure 3. RSF values of active elements in cathodes of $\mathrm{Li}_{x} \mathrm{Ni}_{a} \mathrm{Co}_{b} \mathrm{Mn}_{c} \mathrm{O}_{2}$ with varying $\mathrm{x}$. 